\title{
Non-conservative instabilities in optical vacuum traps
}

Svak, V., Arita, Y., Simpson, S., Brzobohatý, O., Šiler, M., et al.

V. Svak, Y. Arita, S. H. Simpson, O. Brzobohatý, M. Šiler, P. Jákl, J. Kaňka, P. Zemánek, K. Dholakia, "Non-conservative instabilities in optical vacuum traps," Proc. SPIE 11297, Complex Light and Optical Forces XIV, 112970F (24 February 2020); doi: 10.1117/12.2545948

SPIE. Event: SPIE OPTO, 2020, San Francisco, California, United States 


\title{
Non-conservative instabilities in optical vacuum traps
}

\author{
V. Svak a, Y. Arita ${ }^{b}$, S. H. Simpsonª , O. Brzobohatýa, M. Šilerª, P. Jákl ${ }^{a}$, J. Kaňka a , P. Zemánekª \\ and K. Dholakia ${ }^{\mathrm{b}}$ \\ ${ }^{a}$ The Czech Academy of Sciences, Institute of Scientific Instruments, Královopolská 147, 612 \\ 64 Brno, Czech Republic

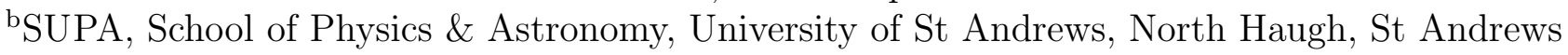 \\ KY16 9SS, United Kingdom
}

\begin{abstract}
Particles held in optical tweezers are commonly thought to be at thermodynamic equilibrium with their environment. Under this assumption the elastic energy of the trap is equal to the thermal energy. As a result the variance of the particle position is completely independent of viscosity and inversely proportional to the optical power in the trap. Here we show that these conditions only hold for very high symmetry cases e.g. perfectly spherical particles in unaberrated, linearly polarized Gaussian traps. Here we show that any reduction in symmetry leads to asymmetrically coupled degrees of freedom. The associated force field is linearly non-conservative and the tweezer is no longer at equilibrium. In overdamped systems the effect is a underlying systematic bias to the Brownian motion. In underdamped systems, this systematic component can accumulate momentum, eventually destabilizing the trap. We illustrate this latter effect with reference to two systems, (i) an isotropic sphere in a circularly polarized trap, and (ii) a birefringent sphere in a linearly polarized trap. In both cases the instability can be approached either by decreasing air pressure or by increasing optical power. Close to instability, the trapped particle executes increasingly coherent motion that is highly sensitive to external perturbations. Potential applications to weak force sensing are discussed.
\end{abstract}

Keywords: Optical force, spin, birefringence, instability

\section{INTRODUCTION}

Optical tweezers are invaluable tools in the meso-scopic sciences. They can be used to hold and move particles with dimensions ranging from nanometres to microns. In addition, they can be used measure forces in the piconewton range, making the instrument ideal for probing the mechanics of microbiological entities and other systems of interest. ${ }^{1}$ Both of these capacities derive from the assumption that the optical forces imposed by the tweezer are approximately linear for small displacements and that they are conservative. With these two assumptions we can calibrate the tweezer by comparing the elastic energy to the thermal energy e.g. ${ }^{1} k_{B} T=$ $\frac{1}{2} P k\left\langle x^{2}\right\rangle$, where $P$ is the optical power, $x$ is the position and $\left\langle x^{2}\right\rangle$ its variance. ${ }^{2-5}$ The trap stiffness for unit power is $k$, so that the optical force, $f^{o p t}=-k x$, and $\left\langle x^{2}\right\rangle$. By measuring the position variance, $\left\langle x^{2}\right\rangle$ we can estimate the stiffness, $k$, allowing us to connect force to measurements of displacement. Importantly, $\left\langle x^{2}\right\rangle$ is inversely proportional to the optical power, $P$ i.e. the harder the particle is held, the less it moves.

$$
\left\langle x^{2}\right\rangle=\frac{k_{B} T}{P k}
$$

In this article, we show that these very useful properties are restricted to high symmetry systems, the most common of which is an isotropic, non-absorbing, perfectly spherical particle in an unaberrated, linearly polarized Gaussian beam. Any departure from this symmetry will, in general, induce linear coupling between degrees

Further author information: (Send correspondence to S.H.S.)

S.H.S.: E-mail: simpson@isibrno.cz

Y.A.: E-mail: ya10@st-andrews.ac.uk 
of freedom and this coupling will cause the optical forces to be linearly non-conservative. Consider $x$ and $y$ coordinates, for example. In the absence of coupling, the corresponding forces are:

$$
\begin{aligned}
& f_{x}^{o p t}=-P k_{x x} x, \\
& f_{y}^{o p t}=-P k_{y y} y
\end{aligned}
$$

where $f_{x}^{o p t}$ and $f_{y}^{o p t}$ are the optical forces in the $x$ and $y$ directions, and $k_{x x}, k_{y y}$ are the stiffness coefficients per unit power, $P$, in the $x$ and $y$ directions. Because they are uncoupled, these forces are effectively one dimensional for small displacements. A one dimensional force field is necessarily conservative because it can always be expressed as the derivative of a scalar potential. However, if there is an appropriate reduction in symmetry, due to modifications either in the trapping beam or the shape of the particle, then additional coupling coefficients appear, connecting motion in the $x$ and $y$ directions:

$$
\begin{aligned}
& f_{x}^{o p t}=-P\left(k_{x x} x+k_{x y} y\right), \\
& f_{y}^{o p t}=-P\left(k_{y x} x+k_{y y} y\right),
\end{aligned}
$$

This constitutes a fundamental difference. When $x$ and $y$ are coupled the coefficients $k_{x y}$ and $k_{y x}$ are not necessarily the same. Whenever they are not the same, the force is linearly non-conservative and a sequence of $x$ and $y$ displacements which return the particle to its initial position will be associated with a transfer of energy between the optical field and the particle. ${ }^{6-8}$ This linear non-conservatism is connected with the fact that light is a flow of momentum and the forces it induces are not generally derivable from a scalar potential. In overdamped systems (i.e. optical trapping in water) the consequences of linear non-conservative motion are quite subtle. The variance of the particle coordinate does not change measurably. However, time correlations reveal that the Brownian motion of the particle contains an underlying, systematic bias. These effects have been described theoretically and measured experimentally. ${ }^{6,9,10}$ In the underdamped regime linear non-conservatism has a more dramatic effect. ${ }^{11}$ In particular, quasi-periodic paths that increase the energy of the particle accumulate momentum and grow in amplitude while ones that lose energy are suppressed. If the optical forces are sufficiently high, or if the viscous drag is low enough then some of the trajectories grow so much that they exceed the linear range of the trap. Beyond this, the particle may execute closed trajectories, possibly limit cycles, that rely on non-linearities in the force field. If viscous damping is further reduced or optical forces further increased then inertial forces overcome optical forces and the particle escapes from the trap.

In the following article we consider two examples of the general behaviour discussed above. In the first example, we consider the motion of an isotropic, non-absorbing silica sphere in a circularly polarized trap. Here the circular polarization induces small transverse forces that tend to promote orbital motion. Initially, the centripetal forces experienced by the particle are smaller than the gradient forces. However, as the power is increased, angular momentum builds up and eventually the particle begins to execute regular, coherent orbits. These orbits are limit cycles and rely on non-linearities in the force. For yet higher powers the particle is thrown from the trap. The second example features a birefringent sphere in a linearly polarized Gaussian trap. In this case, the symmetry is broken by the anisotropy of the refractive index. Rotational degrees of freedom couple to translational motion producing linear oscillations whose amplitude grows as the air pressure, and therefore the viscous damping, is reduced. Eventually the particle is ejected from the trap.

\section{TRANSVERSE SPIN FORCES}

Inhomogeneous optical spin (i.e. circular polarizarion) is associated with a controversial component of optical momentum. ${ }^{12}$ It is a generic feature of structured light fields and has been directly measured in circularly polarized evanescent waves, just above surfaces, and near-paraxial Gaussian beams, in free space. ${ }^{12,13}$ Fig. (1) shows a vector plot of the transverse components of the Poynting vector in a weakly focused, circularly polarized, counter-propagating Gaussian beam. This component of the Poynting vector is almost exclusively associated with inhomogeneous spin. Consequently it is azimuthally directed, circulating about the beam axis. The force 


\section{Transverse force and momentum}

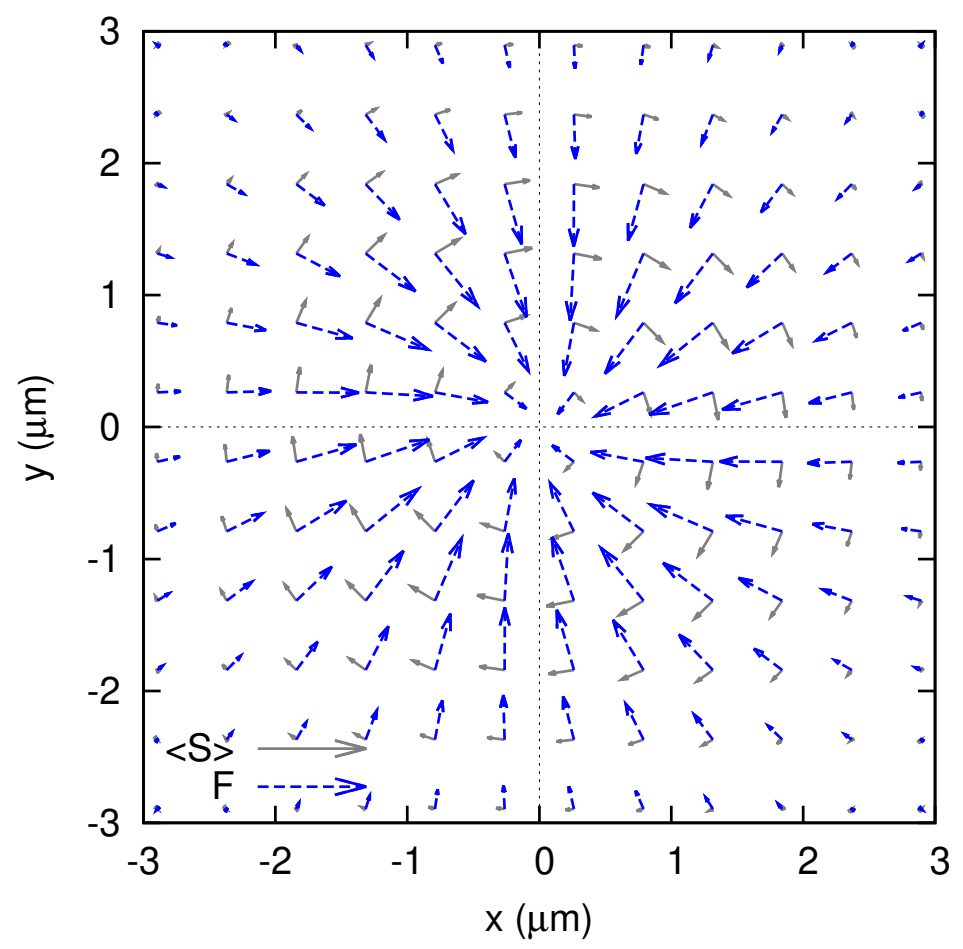

Figure 1. Vector field plot of the transverse components of the Poynting vector and optical force

vector acting on a microsphere is also shown. It is dominated by the gradient force and is therefore directed toward the beam axis. However, the azimuthal spin momentum imparts a weak twist to the force.

The subtly twisted optical force effectively couples the $x$ and $y$ components of the optical force. In moderate vacuum, this influences the thermal motion as follows. For low beam powers this coupling has little effect and the trap behaves conventionally and the probability distribution of the particle coordinates is approximately Gaussian. In this regime, any angular momentum acquired by the particle is effectively dissipated into the ambient gas preventing it from accumulating. As the power is increased, angular momentum starts to build up and, as it does so, centripetal forces grow, pushing the particle outwards against the optical gradient forces. The Gaussian probability distribution of the particle coordinates spreads out. This is the complete opposite of the conventional behaviour, discussed in the introduction, in which increases in power cause the particle to be more tightly bound in the trap. Until now, the particle has optical force field encountered by the particle is approximately linear, so limit cycles (or orbits) are prohibited. However, if the power is further increased, it reaches a threshold in which the centripetal forces overcome optical gradient forces. The particle is now pushed beyond the linear range of the trap. The non-linearities it now experiences allow for the existence of limit cycles and the particle starts to execute stable orbits in which the outward centripetal forces exactly balance the inward gradient forces. This motion is highly coherent. Figure (2) shows experimental results. Red and blue points show the time averaged radial coordinate as the power is increased and decreased, respectively. The small, repeatable hysteresis is due to the asymmetric stability profile of the orbits. Black points show the behaviour in a linearly polarized trap, where transverse spin momentum is absent. Under these conditions, the trap behaves normally. The plots below the graph show the distribution of the centre of mass with optical power increasing from left to right. The top row of figures correspond to increasing power, the lower row to decreasing power.

In this experiment, the non-conservative instability acts as a sensitive measure of azimuthal force, inflating quit small forces into a very large increases in the amplitude of the motion. In this case, the azimuthal force is a consequence of inhomogeneous spin and differs fundamentally from the more familiar components of orbital momentum. ${ }^{14}$ 

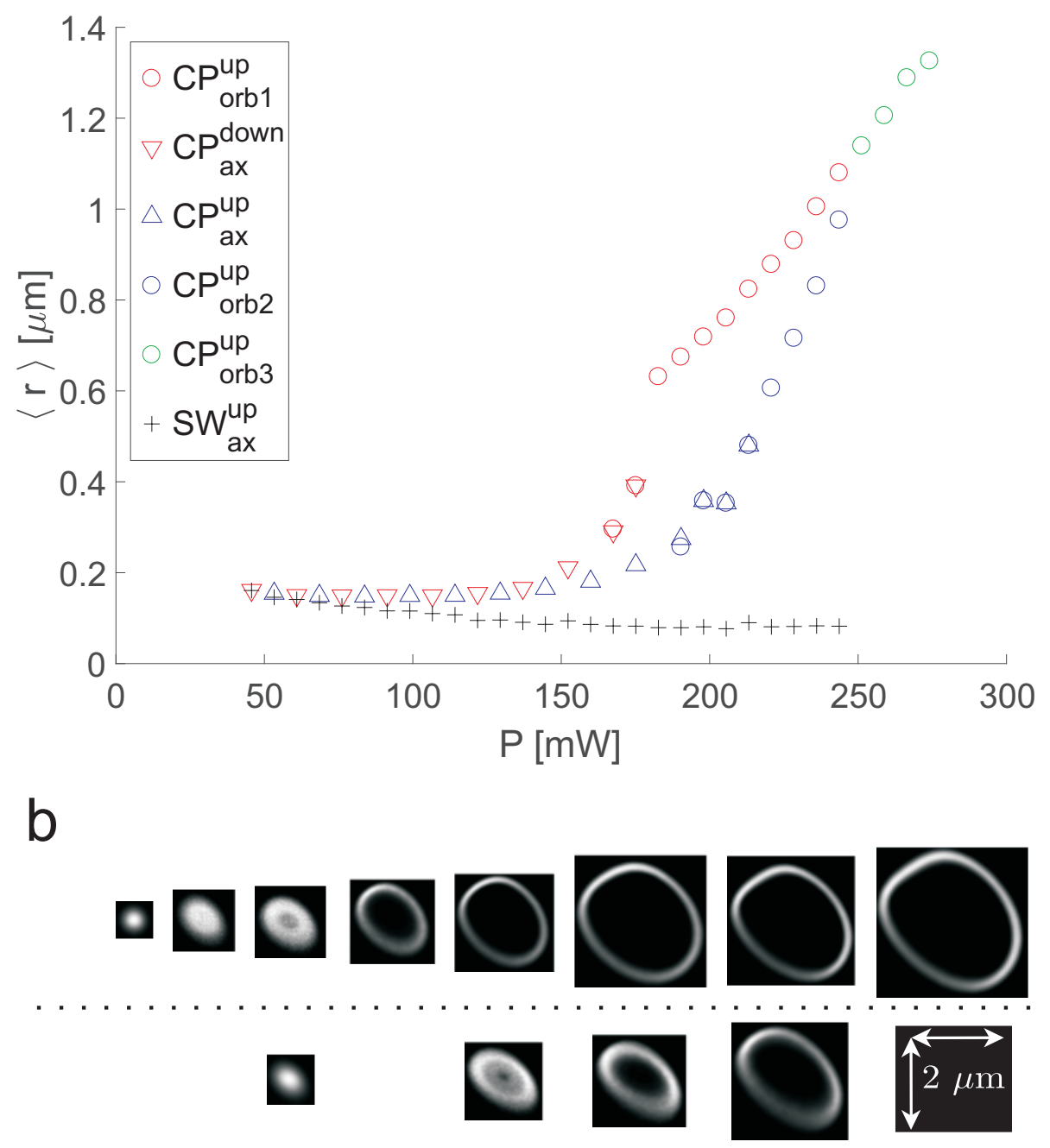

Figure 2. A label of "Video/Audio 1, 2, . ." should appear at the beginning of the caption to indicate to which multimedia file it is linked. Include this text at the end of the caption: http://dx.doi .org/10.1117/12.2545948.1

\section{COHERENT OSCILLATIONS OF BIREFRINGENT SPHERES}

In this second system, we consider the thermal motion of birefringent vaterite spheres held in a linearly polarized vacuum trap. Previous experimental work with vaterite has concentrated on the motion in circularly polarized light fields, where the particles spin fast enough to be influenced by gyroscopic effects. ${ }^{15-17}$ In linearly polarized light the the birefringent vaterite particles align with the polarization. When they are displaced along this direction, they experience a restoring force. In addition, they feel a coupling torque about the transverse axis normal to the polarization. Similarly, if they are rotated about this same axis they feel, in addition to a restoring torque, a coupling force parallel to the polarization. In this way, rotations and translations are coupled, see Fig.(3).

When the particle is trapped in a weak vacuum, its motion is conventional, satisfying equipartition, as described in the introduction. As the pressure is reduced, the non-conservative effects caused by the asymmetric rotation-translation coupling manifest themselves and the amplitude of the translational motion parallel to the 

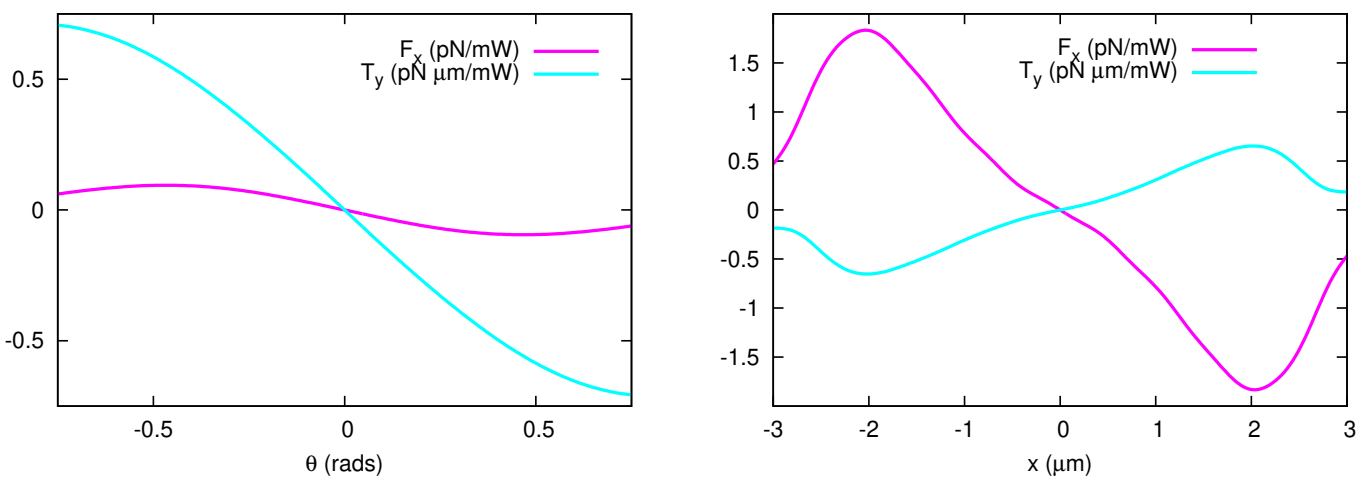

Figure 3. Forces and torques acting on optically trapped vaterite. Left hand side: forces and torques induced by rotations about an axis normal to the polarization. Right hand side: forces and torques induced by displacements parallel to the polarization.

polarization begins to grow. As it does so, the corresponding peak in the power spectrum narrows and grows in height, signifying the emergence of a highly coherent oscillation. This can also be appreciated by considering the relaxation time for the trapped particle, as described by the time correlations. These features are summarized in Fig. 4, below.
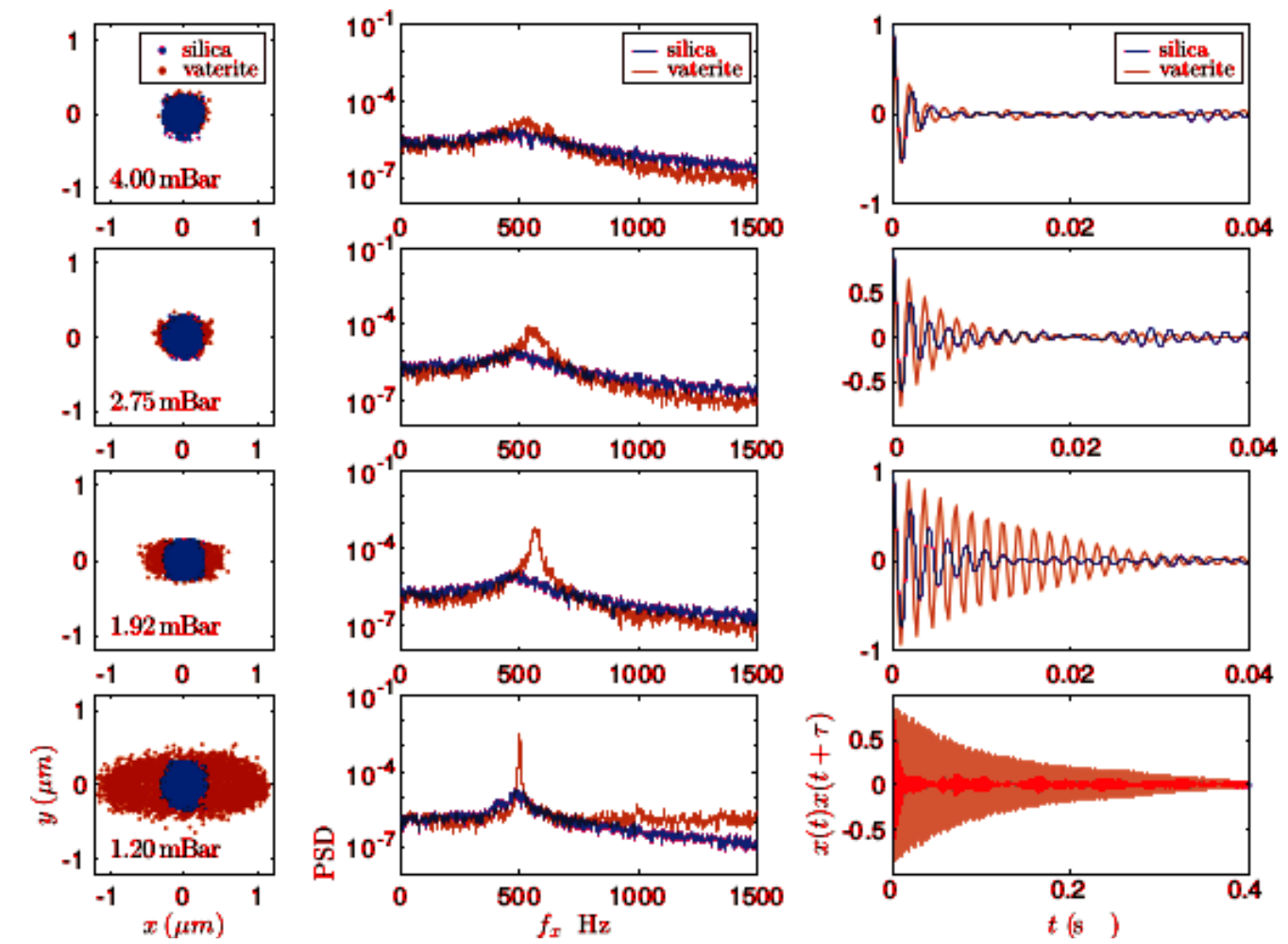

Figure 4. Thermal motion of an optically trapped vaterite sphere in a linearly polarized Gaussian beam in vacuum. Each row relates to a successively lower pressure. The left hand column shows a scatter plot of the $x$ and $y$ coordinates. The middle column shows power spectral densities and the right hand column gives auto-correlation functions for the $x$ coordinate. 


\section{ACKNOWLEDGMENTS}

We acknowledge the support from Engineering and Physical Sciences Research Council (EP/030017/1) and Czech Science Agency (GA19-17765S).

\section{REFERENCES}

[1] Phillips, D. B., Padgett, M. J., Hanna, S., Ho, Y. L. D., Carberry, D. M., Miles, M. J., and Simpson, S. H., "Shape-induced force fields in optical trapping," Nat. Photon. 8, 400-405 (MAY 2014).

[2] Simpson, S. H. and Hanna, S., "Thermal motion of a holographically trapped spm-like probe," NANOTECHNOLOGY 20 (SEP 30 2009).

[3] Phillips, D. B., Gibson, G. M., Bowman, R., Padgett, M. J., Hanna, S., Carberry, D. M., Miles, M. J., and Simpson, S. H., "An optically actuated surface scanning probe," OPTICS EXPRESS 20, 29679-29693 (DEC 31 2012).

[4] Phillips, D. B., Simpson, S. H., Grieve, J. A., Bowman, R., Gibson, G. M., Padgett, M. J., Rarity, J. G., Hanna, S., Miles, M. J., and Carberry, D. M., "Force sensing with a shaped dielectric micro-tool," EPL 99 (SEP 2012).

[5] Phillips, D. B., Simpson, S. H., Grieve, J. A., Gibson, G. M., Bowman, R., Padgett, M. J., Miles, M. J., and Carberry, D. M., "Position clamping of optically trapped microscopic non-spherical probes," OPTICS EXPRESS 19, 20622-20627 (OCT 10 2011).

[6] Simpson, S. H. and Hanna, S., "First-order nonconservative motion of optically trapped nonspherical particles," Phys. Rev. E 82, 031141 (SEP 30 2010).

[7] Simpson, S. H. and Hanna, S., "Stability analysis and thermal motion of optically trapped nanowires," Nanotech. 23 (MAY 2012).

[8] Simpson, S. H. and Hanna, S., "Holographic optical trapping of microrods and nanowires," J. Opt. Soc. Am. A 27, 1255-1264 (JUN 2010).

[9] Irrera, A., Magazzú, A., Artoni, P., Simpson, S. H., Hanna, S., Jones, P. H., Priolo, F., Gucciardi, P. G., and Maragó, O. M., "Photonic torque microscopy of the nonconservative force field for optically trapped silicon nanowires," Nano Lett. 16, 4181-4188 (JUL 2016).

[10] Toe, W. J., Ortega-Piwonka, I., Angstmann, C. N., Gao, Q., Tan, H. H., Jagadish, C., Henry, B. I., and Reece, P. J., "Nonconservative dynamics of optically trapped high-aspect-ratio nanowires," Phys. Rev. E 93, 022137 (FEB 24 2016).

[11] Svak, V., Brzobohatỳ, O., Šiler, M., Jákl, P., Kaňka, J., Zemánek, P., and Simpson, S., "Transverse spin forces and non-equilibrium particle dynamics in a circularly polarized vacuum optical trap," Nat. Commun. 9(1), 5453 (2018).

[12] Bliokh, K. Y., Bekshaev, A. Y., and Nori, F., "Extraordinary momentum and spin in evanescent waves," Nat. Commun. 5, 3300 (MAR 2014).

[13] Antognozzi, M., Bermingham, C. R., Harniman, R. L., Simpson, S., Senior, J., Hayward, R., Hoerber, H., Dennis, M. R., Bekshaev, A. Y., Bliokh, K. Y., and Nori, F., "Direct measurements of the extraordinary optical momentum and transverse spin-dependent force using a nano-cantilever," Nat. Phys. 12, 731-735 (AUG 2016).

[14] Arita, Y., Chen, M., Wright, E. M., and Dholakia, K., "Dynamics of a levitated microparticle in vacuum trapped by a perfect vortex beam: three-dimensional motion around a complex optical potential," J. Opt. Soc. Am. B 34(6), C14-C19 (2017).

[15] Arita, Y., Mazilu, M., and Dholakia, K., "Laser-induced rotation and cooling of a trapped microgyroscope in vacuum," Nat. Commun. 4, 2374 (2013).

[16] Arita, Y., McKinley, A. W., Mazilu, M., Rubinsztein-Dunlop, H., and Dholakia, K., "Picoliter rheology of gaseous media using a rotating optically trapped birefringent microparticle," Anal. Chem. 83, 8855-8858 (2011).

[17] Arita, Y., Wright, E. M., and Dholakia, K., "Optical binding of two cooled micro-gyroscopes levitated in vacuum," Optica 5(8), 910-917 (2018). 